REVISTA CIENCIAS BIOMÉ DICAS

ARTÍCULOS ORIGINALES

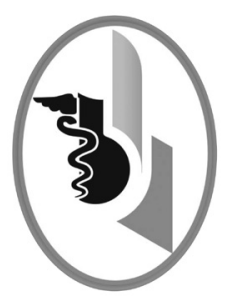

\title{
CUIDADO SEGURO POR ENFERMERÍA EN EL HOSPITAL INFANTIL NAPOLEÓN FRANCO PAREJA EN CARTAGENA
}

\author{
PATIENT SAFETY BY NURSING IN THE HOSPITAL INFANTIL \\ NAPOLEÓN FRANCO PAREJA IN CARTAGENA
}

\author{
Romero-Massa Elizabeth ${ }^{1}$ \\ Howard-Bernard Kadine Savija ${ }^{2}$ \\ Martínez-Julio Tania Andrea²
}

Correspondencia: eromerom@unicartagena.edu.co

Recibido para evaluación: septiembre 25 - 2016. Aceptado para publicación: noviembre - 1 - 2016

\section{RESUMEN}

Introducción: la seguridad del paciente implica la evaluación permanente y proactiva de los riesgos asociados a la atención en salud.

Objetivo: describir las prácticas de cuidado seguro de las enfermeras en el Hospital Infantil Napoleón Franco Pareja.

Materiales y métodos: estudio descriptivo. La población y muestra contó con un total de 60 enfermeras y se aplicaron los instrumentos GCTC_UN-PROF y "Percepción de seguridad de pacientes de los profesionales". Mediante estadística descriptiva los datos se presentaron en frecuencias y promedios.

Resultados: predominó el género femenino en $98.3 \%$, con una edad promedio de 34 años y experiencia laboral de 9 años. En relación a las prácticas de cuidado seguro, se observó que la política de seguridad del paciente por las enfermeras se aplica en un $100 \%$. Estas afirmaron conocer los eventos adversos de reporte obligatorio y los indicadores de seguimiento de cuidado seguro en el $91.7 \%$ y los monitorearon en el $90.0 \%$. Asimismo, conocen y toman medidas preventivas para mitigar los riesgos para el paciente en un $95.0 \%$. En cuanto a la percepción de seguridad del paciente los enfermera/os con menores promedios siempre sienten que su participación es activa en la consecución de los objetivos de seguridad del paciente con $33.8 \%$ y casi siempre $32.5 \%$. En menor grado, las enfermeras siempre perciben autonomía y delegación de responsabilidad en los objetivos del programa en un $20 \%$. Sobre fomento a la investigación obtuvieron un $20 \%$ en la categoría siempre, a veces con un $25 \%$ y casi nunca $21.7 \%$.

1 Enfermera. Magíster en Enfermería. Profesor Titular. Universidad de Cartagena. Cartagena. Colombia.

2 Enfermera. Universidad de Cartagena. Cartagena. Colombia. 
Conclusión: el cuidado seguro en enfermería respondió a los lineamientos vigentes y se caracterizó por buenas prácticas del mismo. Es necesario fortalecer la participación con autonomía en los procesos y la investigación. Rev.cienc.biomed. 2016;7(2): 249-257.

PALABRAS CLAVE

Cuidado del niño; Seguridad del paciente; Enfermería.

\section{SUMMARY}

Introduction: patient safety involves permanent and proactive assessment of the risks associated with health care.

Objective: to describe the safe care of nurses at Hospital Infantil Napoleón Franco Pareja.

Materials and methods: a descriptive cross-sectional study was carried out. The total of the sample was 60 nurses. The instruments GCTC_UN-PROF and "Perception of patient safety professionals" were applied. Descriptive statistics data are presented in frequencies and averages.

Results: female gender was predominance in 98.3\%; the average age was 34 years with a work experience of 9 years. In relation to the practices of safe care, it was observed that the policy of patient safety by nurses was applied in $100 \%$. They affirmed to know the adverse events of mandatory reporting and monitoring indicators care insurance $91.7 \%$ that were followed for them in $90.0 \%$. Also, the nurses know and take preventive measures to mitigate the risks to the patient in $95.0 \%$. As for the perception of patient safety, nurses with lower average always feel recognized for their active participation in achieving the objectives of patient safety with a percentage of $33.8 \%$; almost always in $32.5 \%$. To a lesser degree, nurses always perceived autonomy and delegation of responsibility to the program objectives with $20 \%$. According to the research, the nurses got a $20 \%$ in always; sometimes with $25 \%$ and almost never with $21.7 \%$.

Conclusion: patient safety by nursing responds to current guidelines, characterized by good safe care practices. It is necessary to strengthen participation with autonomy and research. Rev.cienc.biomed. 2016;7(2):249-257.

\section{KEYWORDS}

Child Care; Patient safety; Nursing.

\section{INTRODUCCIÓN}

El individuo en su etapa inicial de desarrollo se encuentra más vulnerable a sufrir lesiones durante la estancia hospitalaria, por lo que existe la necesidad de contar con medidas de seguridad específicas para evitar riesgos durante su cuidado (1). Para proporcionar cuidado seguro, establecer garantía de calidad y prevenir los errores es necesario establecer un mecanismo de información que permita a los gestores de enfermería determinar la existencia de focos rojos y la posibilidad de controlarlos, disminuirlos 0 desparecerlos (2).

La estrategia de seguridad del paciente que desarrolla Colombia es el conjunto de acciones y estrategias sencillas que está impulsando el Ministerio de la Protección
Social para que sean implementadas por las instituciones prestadoras de servicios de salud. Con ellas se pretende ofrecer herramientas prácticas para aumentar la seguridad de los procesos de atención en salud, impactar en la mejora de la calidad y proteger al paciente de riesgos evitables (3).

El concepto seguridad del paciente es entendido como la estructura, los procesos, los instrumentos y las metodologías basadas en evidencia científica, cuya finalidad es coordinar esfuerzos para disminuir el riesgo de sufrir un daño prevenible en el proceso de atención de salud o de atenuar sus consecuencias (4).

En 2004, la Organización Mundial de Salud (OMS) creó el proyecto de Alianza Mundial para la Seguridad del Paciente, cuyo objetivo 
fundamental fue prevenir daños en estos últimos. Datos de investigación obtenidos de los Estados Unidos en 1999 indican que los errores en el cuidado de la salud causan aproximadamente entre 44.000 y 98.000 eventos adversos anualmente en los hospitales de aquel país (5).

En la actualidad, Colombia impulsa una política de seguridad del paciente, liderada por el Sistema Obligatorio de Garantía de Calidad de la Atención en Salud, establecida en la Resolución 1446 de 2006 y cuyo objetivo es prevenir la ocurrencia de situaciones que afecten la seguridad del paciente, reducir y de ser posible, eliminar la ocurrencia de eventos adversos para contar con instituciones seguras y competitivas internacionalmente (6). La Resolución 1441 de 2013 define que las instituciones en salud deben implementar el Programa de Seguridad del Paciente con cuatro elementos fundamentales: planeación estratégica de la seguridad del paciente, fortalecimiento de la cultura institucional, medición, análisis, reporte y gestión de los eventos adversos y procesos seguros (7).

Mejorar la seguridad del paciente es un objetivo global, puesto que errores en la administración de medicamentos contribuyen significativamente en la alta tasa de eventos adversos que ocurren anualmente en los hospitales. Los eventos adversos son frecuentemente de alto costo y generan daños en los pacientes, a los profesionales de la salud y a los hospitales. Muchas iniciativas que enfocan la seguridad del paciente han sido tomadas en todo el mundo, pero aún se precisan más investigaciones para determinar intervenciones de costo más efectivas en aras de crear sistemas más seguros para el cuidado del paciente (8).

Modelski (9) expresa que un niño por su fragilidad, vulnerabilidad y condiciones peculiares de crecimiento y desarrollo necesita protección, atención especial y cuidado seguro por parte de enfermería. El profesional de Enfermería está Ilamado a hacer parte, a nivel personal e institucional, de las iniciativas que buscan estudiar y disminuir el impacto negativo de los eventos adversos en los sistemas de salud.
En un estudio realizado por el área de enfermería, en Brasil, se encontró 2.9 de ocurrencia de eventos adversos en niños como resultado de prácticas indebidas en dicha área. Los eventos relacionados con la administración de medicamentos fueron los más frecuentes $(32.7 \%)$. Seguidos por la ventilación mecánica aérea $(29.2 \%)$, procedimientos de enfermería $(16.8 \%)$, manejo de catéteres, sondas y drenajes $(14.3 \%)$, manejo de equipos (4.4\%) $y$ otros $(2.6 \%)(10)$.

Especialmente en pediatría, los eventos adversos deben ser reconocidos y discutidos por la vulnerabilidad y dependencia de estos pacientes a la calidad de los servicios de salud (11). Brindar cuidados seguros en niños responde a un modo de actuación del profesional de Enfermería, elemento esencial en la cultura de calidad que se estampa en los servicios de salud (12).

Para la enfermería es una necesidad realizar estudios que proporcionen un alto nivel de evidencia científica, que permitan documentar las condiciones y las causas que favorecen o que ponen en riesgo un ambiente de seguridad hacia la promoción de un cuidado de enfermería seguro. La relevancia del presente estudio radica en describir el cuidado seguro brindado por enfermeras en el Hospital Infantil Napoleón Franco Pareja.

\section{MATERIALES Y MÉTODOS}

Estudio descriptivo de corte transversal. La población y muestra la constituyó un total de 60 enfermeras profesionales del Hospital Infantil Napoleón Franco Pareja, en la ciudad de Cartagena, en el primer período del año 2016, que cumplieron los criterios de inclusión de permanecer por más de un año vinculadas al hospital y que estuvieran laborando en los servicios de urgencias, quirófanos, hospitalización, UCIN, UCI pediátrica, Unidad de Cuidados Cardiovasculares, Unidad Oncológica, y que desearan participar de manera voluntaria en el estudio.

Una vez se tuvo la aprobación de la institución donde se realizó la investigación, se identificó con el listado las/os enfermeras (os) de los diferentes servicios para proceder a diligenciar el consentimiento informado y 
socializar los objetivos de la investigación a los participantes, a los cuales se les aplicaron las encuestas después de la salida de los turnos.

La aplicación de la encuesta de práctica de cuidado seguro fue diseñada por el grupo de Cuidado al paciente crónico y su familia de la Universidad Nacional de Colombia en el instrumento GCPC_UN- PROF (13). En una primera parte, el instrumento permite caracterizar y conocer la experiencia profesional de las enfermeras, contiene 10 preguntas cerradas. En un segundo apartado, sobre Práctica de cuidado seguro por enfermería, contiene 8 preguntas cerradas con opción de respuesta afirmativa o negativa, y abierta. Esta herramienta posee pruebas de validez y psicométricas desarrolladas por la Universidad Nacional de Colombia (13).

El instrumento denominado "Percepción de seguridad de pacientes por los profesionales", mediante el modelo de estándares de calidad de cuidados para la seguridad del paciente en los hospitales, contó con un grado de fiabilidad en que la concordancia fue superior al 95\% y el índice de Kappa superior a 0.5. Las pruebas estadísticas a las que han sido sometidos los cuestionarios de percepción y el propio modelo, y el hecho de haber sido pilotado en cinco hospitales hace pensar que se presenta una herramienta válida, fiable y viable (14). Consta de 14 ítems que evaluaron tres aspectos: formación del profesional, participación y la seguridad del paciente. De acuerdo con los estándares de calidad en seguridad del paciente cada ítem tuvo una declaración corta acerca de cómo la enfermería percibe la seguridad del paciente en el hospital. El patrón de respuesta está dado por un formato Likert con cinco criterios: nunca, casi nunca, a veces, casi siempre y siempre

El análisis de la información se desarrolló con el empleo del programa SPSS versión 2012, bajo licencia de la Universidad Nacional de Colombia. Mediante estadística descriptiva se expresaron los datos en frecuencias absolutas y relativas; para las variables cualitativas se utilizaron las medidas de tendencia central.

Esta investigación se llevó a cabo teniendo en cuenta, durante todo el desarrollo del es- tudio, la normatividad presente en Colombia y a nivel mundial para la realización de investigaciones en seres humanos como la Declaración de Helsinki (15). La investigación está clasificada en riesgo bajo de acuerdo a la Resolución No 008430 de 1993 del Ministerio de Protección Social de Colombia. Se realizó el diligenciamiento del consentimiento informado a los participantes (16).

\section{RESULTADOS}

El género con mayor predominio fue el femenino con un porcentaje del $98.3 \%$, el género masculino solo contó con un $1.7 \%$. El promedio de edad encontrado fue 34 años y el promedio de experiencia laboral 9 años. Con respecto al estado civil, la mayor parte de la población vivía en unión libre $31.7 \%$. El nivel educativo de enfermeros con diplomado fue $23.3 \%$, especialización $16.7 \%$ y pregrado $60 \%$. Con respecto al servicio en donde laboraban, la mayor cantidad de enfermeras estaban en la UCI, 40\%. El $51.7 \%$ trabajaba mediante contrato a término fijo y el $13.3 \%$ por orden de servicios.

Prácticas del cuidado seguro por enfermería: el cuerpo de enfermería manifestó que aplicaba la política del Ministerio de Salud sobre seguridad del paciente en el Hospital Infantil Napoleón Franco Pareja, en un 95\%, así como las políticas del Sistema Obligatorio de Garantía de la Calidad. El $91.7 \%$ de enfermeras conocía los indicadores de seguimiento de cuidado seguro en su institución, asimismo, la mayoría de ellas respondieron que conocían los eventos adversos de reporte obligatorio en la institución en un $91.7 \%$. En cuanto a la monitorización de los indicadores de cuidado seguro, las enfermeras respondieron afirmativamente con un porcentaje del $90 \%$; el $95 \%$ respondió que tomaban medidas preventivas para mitigar los riesgos del paciente. Todas afirmaron haber reportado los eventos adversos ocurridos. En relación a la capacitación, el $86.7 \%$ contestó que recibió educación a cerca del tema (Tabla No 1).

Percepción de seguridad del paciente por enfermeras: se encontró que el $45.0 \%$ siempre y el $48.3 \%$ casi siempre disponían de estructuras y mecanismos de participación en los objetivos de seguridad de paciente. 


\begin{tabular}{|l|c|c|c|c|}
\hline \multicolumn{4}{|c|}{ TABLA No 1. } \\
\hline \multicolumn{4}{|c|}{ PRÁCTICAS DE CUIDADO SEGURO } \\
\hline $\begin{array}{l}\text { Aplicación de la política del Ministerio de Salud de seguridad del } \\
\text { paciente }\end{array}$ & 60 & 100 & 0 & 0.0 \\
\hline Aplica el sistema obligatorio de garantía de calidad & 57 & 95 & 3 & 5.0 \\
\hline Conoce los indicadores de seguimiento del cuidado seguro obligatorio & 55 & 91.7 & 5 & 8.3 \\
\hline Conoce los eventos adversos de reporte obligatorio & 55 & 91.7 & 5 & 8.3 \\
\hline Monitorea los indicadores de cuidado seguro & 54 & 90 & 6 & 10.0 \\
\hline $\begin{array}{l}\text { Toma medidas preventivas para mitigar los riesgos para el paciente } \\
\text { en el marco del cuidado seguro }\end{array}$ & 57 & 95 & 3 & 5.0 \\
\hline $\begin{array}{l}\text { ¿Reporta institucionalmente los eventos adversos ocurridos en su } \\
\text { labor profesional? }\end{array}$ & 60 & 100 & 0 & 0 \\
\hline ¿Ha recibido capacitación en el tema de cuidado seguro? & 52 & 86.7 & 8 & 13.3 \\
\hline
\end{tabular}

El personal se siente reconocido por su participación activa en la consecución de los objetivos de seguridad del paciente: siempre $33.8 \%$ y casi siempre $32.5 \%$. Con respecto a la percepción de autonomía y delegación de responsabilidad en temas relacionados con la seguridad del paciente se observó lo siguiente: casi siempre $45 \%$, siempre $20 \%$. La mayoría de las enfermeras cuando comete un error, el supervisor(a) o el propio personal llevaban a cabo las medidas correctivas pertinentes: $48.3 \%$ siempre y $38.3 \%$ casi siempre. El $61.7 \%$ de las enfermeras manifestaron sentirse apoyadas siempre por su superior(a) en temas relacionados con la seguridad de los pacientes. Percepción del fomento a la investigación sobre seguridad de los cuidados de los pacientes: casi siempre $28.3 \%$ y siempre $25 \%$ (Tabla No 2 ).

\begin{tabular}{|c|c|c|c|c|c|c|c|c|c|c|}
\hline \multicolumn{11}{|c|}{$\begin{array}{c}\text { TABLA N02. } \\
\text { PERCEPCIÓN DE SEGURIDAD DEL PACIENTE }\end{array}$} \\
\hline & \multicolumn{2}{|c|}{ NUNCA } & \multicolumn{2}{|c|}{$\begin{array}{c}\text { CASI } \\
\text { NUNCA }\end{array}$} & \multicolumn{2}{|c|}{ A VECES } & \multicolumn{2}{|c|}{$\begin{array}{c}\text { CASI } \\
\text { SIEMPRE }\end{array}$} & \multicolumn{2}{|c|}{ SIEMPRE } \\
\hline & $n$ & $\%$ & $\mathrm{n}$ & $\%$ & $n$ & $\%$ & $n$ & $\%$ & $\mathrm{n}$ & $\%$ \\
\hline $\begin{array}{l}\text { Los profesionales disponen de } \\
\text { estructuras y mecanismos de } \\
\text { participación en los objetivos de } \\
\text { seguridad del paciente. }\end{array}$ & 0 & 0.0 & 0 & 0.0 & 4 & 6.7 & 29 & 48.3 & 27 & 45.0 \\
\hline $\begin{array}{l}\text { Me siento reconocido(a) por mi } \\
\text { participación activa en la consecución } \\
\text { de los objetivos de seguridad del } \\
\text { paciente. }\end{array}$ & 2 & 2.5 & 1 & 1.3 & 24 & 30.0 & 26 & 32.5 & 27 & 33.8 \\
\hline $\begin{array}{l}\text { Percibo autonomía y delegación de } \\
\text { responsabilidad en temas relacionados } \\
\text { con la seguridad del paciente. }\end{array}$ & 1 & 1.7 & 3 & 5.0 & 17 & 28.3 & 27 & 45.0 & 12 & 20.0 \\
\hline $\begin{array}{l}\text { Cuando se ha cometido un error, el } \\
\text { supervisor(a) y el propio personal } \\
\text { lleva a cabo las medidas correctivas } \\
\text { pertinentes. }\end{array}$ & 0 & 0.0 & 0 & 0.0 & 8 & 13.3 & 23 & 38.3 & 29 & 48.3 \\
\hline $\begin{array}{l}\text { Me siento apoyado(a) por mi } \\
\text { superior(a) en temas relacionados con } \\
\text { la seguridad de los pacientes. }\end{array}$ & 0 & 0.0 & 0 & 0.0 & 6 & 10.0 & 17 & 28.3 & 37 & 61.7 \\
\hline $\begin{array}{l}\text { Se fomenta la investigación sobre } \\
\text { la seguridad de los cuidados de los } \\
\text { pacientes en el hospital. }\end{array}$ & 0 & 0.0 & 13 & 21.7 & 15 & 25.0 & 17 & 28.3 & 15 & 25.0 \\
\hline
\end{tabular}


Se encontró que los profesionales de Enfermería encuestados manifestaron que disponían de canales de comunicación para poder hacer sugerencias sobre temas de seguridad del paciente: $45 \%$ casi siempre y siempre $36.7 \%$. Además, el $45 \%$ de las enfermeras afirmó que casi siempre se pactaron los objetivos específicos anualmente relacionados con la seguridad de los cuidados a los pacientes, mientras que el $43.3 \%$ lo pactaba siempre. En caso de disponer de objetivos de seguridad para los cuidados de los pacientes, el personal de enfermería siempre recibió información $38.3 \%$. En relación al sistema de notificación de incidentes y eventos adversos, el 55\% afirmó que siempre contó con este y
$31.7 \%$, casi siempre. El 50\% manifestó que siempre los sistemas de notificación de incidentes y eventos adversos fueron adecuados (Tabla No 3).

En relación a la formación del profesional a partir de su vinculación a la institución, el $45 \%$ manifestó que el hospital siempre o casi siempre garantizó y fomentó su acceso a programas de formación continua relacionados con la seguridad del paciente. Además de esto, el 50\% manifestó que siempre se disponía de un plan de formación y acogida para profesionales de nueva incorporación que incluía información sobre los riesgos más frecuentes para los pacientes (Tabla No 4).

\begin{tabular}{|c|c|c|c|c|c|c|c|c|c|c|}
\hline \multicolumn{11}{|c|}{$\begin{array}{l}\text { TABLA No } 3 . \\
\text { CANALES DE COMUNICACIÓN PARA BRINDAR SEGURIDAD DEL PACIENTE }\end{array}$} \\
\hline & \multicolumn{2}{|c|}{ NUNCA } & \multicolumn{2}{|c|}{ CASI NUNCA } & \multicolumn{2}{|c|}{ A VECES } & \multicolumn{2}{|c|}{$\begin{array}{c}\text { CASI } \\
\text { SIEMPRE }\end{array}$} & \multicolumn{2}{|c|}{ SIEMPRE } \\
\hline & $\mathrm{n}$ & $\%$ & $\mathrm{n}$ & $\%$ & $\mathrm{n}$ & $\%$ & $n$ & $\%$ & $\mathrm{n}$ & $\%$ \\
\hline $\begin{array}{l}\text { Los profesionales disponen de canales } \\
\text { de comunicación para poder hacer } \\
\text { sugerencias sobre temas de seguridad } \\
\text { del paciente. }\end{array}$ & 0 & 0.0 & 2 & 3.3 & 9 & 15.0 & 27 & 45.0 & 22 & 36.7 \\
\hline $\begin{array}{l}\text { En mi unidad se pactan anualmente } \\
\text { objetivos específicos relacionados } \\
\text { con la seguridad de los cuidados a los } \\
\text { pacientes. Ejemplos: disminución de } \\
\text { infecciones, caídas, flebitis, lesiones por } \\
\text { presión, etc. }\end{array}$ & 0 & 0.0 & 1 & 1.7 & 6 & 10.0 & 27 & 45.0 & 26 & 43.3 \\
\hline $\begin{array}{l}\text { En caso de disponer de objetivos de } \\
\text { seguridad para los cuidados de los } \\
\text { pacientes, recibo información sobre el } \\
\text { grado de consecución de los mismos. }\end{array}$ & 0 & 0.0 & 1 & 1.7 & 9 & 15.0 & 27 & 45.0 & 23 & 38.3 \\
\hline $\begin{array}{l}\text { En el hospital hay sistema de notifica- } \\
\text { ción de incidentes y eventos adversos. }\end{array}$ & 0 & 0.0 & 0 & 0.0 & 8 & 13.3 & 19 & 31.7 & 33 & 55.0 \\
\hline $\begin{array}{l}\text { Los sistemas de notificación de } \\
\text { incidentes y eventos adversos } \\
\text { son adecuados (accesibles, no } \\
\text { sancionatorios y anónimos). }\end{array}$ & 0 & 0.0 & 0 & 0.0 & 5 & 8.3 & 25 & 41.7 & 30 & 50.0 \\
\hline
\end{tabular}

FORMACIÓN EN SEGURIDAD DEL PACIENTE

\begin{tabular}{|l|c|c|c|c|c|c|c|c|c|c|}
\hline & \multicolumn{2}{|c|}{ NUNCA } & \multicolumn{2}{c|}{ CASI NUNCA } & \multicolumn{2}{c|}{ A VECES } & \multicolumn{2}{|c|}{$\begin{array}{c}\text { CASI } \\
\text { SIEMPR }\end{array}$} & \multicolumn{2}{c|}{ SIEMPRE } \\
\cline { 2 - 12 } & $\mathrm{n}$ & $\%$ & $\mathrm{n}$ & $\%$ & $\mathrm{n}$ & $\%$ & $\mathrm{n}$ & $\%$ & $\mathrm{n}$ & $\%$ \\
\hline $\begin{array}{l}\text { El hospital garantiza y fomenta su } \\
\text { acceso a programas de formación } \\
\text { continua relacionados con la seguridad } \\
\text { del paciente. }\end{array}$ & 0 & 0.0 & 0 & 0.0 & 6 & 10.0 & 27 & 45.0 & 27 & 45.0 \\
\hline $\begin{array}{l}\text { El hospital dispone de un plan de for- } \\
\text { mación y acogida para profesionales de } \\
\text { nueva incorporación que incluye infor- } \\
\text { mación sobre los riesgos más frecuen- } \\
\text { tes para los pacientes. }\end{array}$ & 0 & 0.0 & 0 & 0.0 & 6 & 10.0 & 24 & 40.0 & 30 & 50.0 \\
\hline $\begin{array}{l}\text { Estoy satisfecho con la formación que } \\
\text { recibo sobre seguridad del paciente. }\end{array}$ & 0 & 0.0 & 4 & 6.7 & 10 & 16.7 & 26 & 43.3 & 20 & 33.3 \\
\hline
\end{tabular}




\section{DISCUSIÓN}

Se aprecia en los hallazgos encontrados que los participantes reciben formación sobre cuidado seguro, participan en el programa, en el monitoreo de los indicadores, disponen de mecanismos de participación de objetivos y conocen la política de seguridad del paciente, lo que indica que la institución está construyendo la cultura de seguridad. Avanzar hacia una cultura de seguridad del paciente no solo representa una prioridad profesional y moral, sino que también es tangible el impacto financiero que pueda tener dentro de la organización (17).

Diversos estudios que han medido la percepción de la seguridad del paciente en hospitales, han identificado que el personal de enfermería tiene fortalezas en el trabajo que desarrollan en equipo dentro de las unidades/servicio y el apoyo que se brindan calificando a estas dimensiones con el mayor porcentaje de respuestas positivas $(18,19)$. Además, han evaluado positivamente el aprendizaje que les da la organización.

Se encontró predominio del género femenino en los participantes del estudio, datos similares a los reportados por Melendez (20), quien afirmó que existe una fuerte feminización de las tareas de cuidado, encontrando con respecto al sexo que el $91 \%$ (224) son mujeres y un 9\% (23) hombres.

Adicional a esto, el promedio de experiencia laboral fue de 9 años, datos similares se encontraron en el estudio de Castillo (21), en el cual el grupo de enfermeras (60\%) se distribuyó en la franja de los 5-15 años de experiencia.

Las enfermeras en su mayoría tenían un nivel educativo de pregrado, semejante a lo encontrado por Castillo (21), este menciona que el $86.5 \%$ de enfermeras tiene solo el título de pregrado. Esto puede deberse a la poca motivación para continuar estudios de postgrado por la sobrecarga laboral a la que es sometida la enfermera, los extensos turnos rotativos y el poco incentivo monetario en las instituciones.

En este estudio, las enfermeras mostraron fortalezas al responder que siempre $55 \%$ y casi siempre $31.7 \%$ cuentan con un sistema de notificación de incidentes y eventos adversos adecuados y monitorizan indicadores de cuidado seguro. El compromiso con el cuidado seguro le implica a la institución pasar de la vigilancia externa a la propia supervisión del castigo y el control policivo a cargo de otros, al autocontrol sobre los procesos, de eventos adversos a la prevención de los riesgos y de la falta de compromiso o comprensión de algunos, a la proactividad, la participación, la consulta y la retroalimentación a todo el sistema (22).

De acuerdo a las características de la práctica del cuidado seguro se encontró que los enfermeros del hospital infantil, siempre y casi siempre, con iguales promedios en mayor grado, cuentan con una formación profesional en relación a la seguridad del paciente y disponen de un plan de formación en el tema. Cada individuo debe mantener actualizados los diferentes conocimientos que requiere el cuidado para mejorar de manera persistente, eso implica lo personal, lo empírico, lo artístico y, por supuesto, lo ético (22).

Gómez (23), en relación a la seguridad del paciente referida por el personal de enfermería, se evidencia por resultados positivos en la coordinación y comunicación eficaz dentro de las unidades y la percepción generalizada de que la gerencia se interesa en la seguridad del paciente. En los aspectos a mejorar se destaca la percepción de personal insuficiente y el alto flujo de personal ocasional percibidos como riesgos en la atención que afecta la seguridad del paciente.

Difieren de estos resultados los de Barbosa (19), en Colombia 2008, que encontró que el grado de seguridad del paciente entre los encuestados en un hospital universitario, es percibido como deficiente, y concluye "que si existe un aprendizaje organizacional, respecto al análisis de los errores, que lleva a cambios positivos en el manejo de la seguridad del paciente y todos los esfuerzos están orientados a este fin, reconociendo que el mejoramiento continuo se da, pero requiere ser fortalecido en el día a día".

Se encontró que los profesionales encuestados de Enfermería manifestaron en un gran porcentaje que disponían de canales de co- 
municación para poder hacer sugerencias sobre temas de seguridad del paciente. Meyer (24) relacionó la falta de informacióncomunicación por parte del personal sanitario con una mayor preocupación de sufrir un error en la atención. Este aporte permite visualizar que la información realmente es la base de la implementación de una cultura de seguridad del paciente. Organizaciones con una cultura de seguridad positiva se caracterizan por una comunicación abierta y basada en la confianza mutua, gestión del riesgo, por percepciones comunes acerca de la importancia de la seguridad y el aprendizaje organizacional y por la confianza en la eficacia de las medidas preventivas $(25,26)$.

El fomento de la investigación en cuanto a seguridad del paciente por enfermería obtuvo los promedios más bajos en el presente estudio. Al respecto, la Alianza Mundial para la Seguridad del Paciente afirmó que los factores importantes que explican el aún limitado corpus de la investigación sobre la seguridad del paciente destacan la escasa sensibilización y apoyo político y económico, junto al limitado desarrollo metodológico. Uno de los mayores desafíos consiste en fortalecer la capacidad de investigación para abordar mejor las cuestiones de mayor repercusión para la reducción del problema (25).
La participación y el empoderamiento de los pacientes y ciudadanos en su propia seguridad en el hospital han ido cobrando importancia creciente a nivel nacional e internacional. Las enfermeras requieren aplicar procedimientos y prácticas que han demostrado efectividad para reducir la aparición de errores y eventos adversos y también generar nuevos conocimientos sobre los factores que contribuyen a mejorar la seguridad del paciente.

\section{CONCLUSIÓN}

El cuidado seguro brindado por enfermería responde a los lineamientos vigentes, constituyéndose en un proceso institucional, caracterizado por el fomento de la formación y el conocimiento, monitoreo de indicadores, fomento de medidas preventivas, cultura del reporte y canales de comunicación adecuados. Es necesario fortalecer la participación con autonomía en los procesos y en investigaciones.

CONFLICTO DE INTERESES: ninguno que declarar.

FINANCIACIÓN: recursos propios de los autores.

\section{REFERENCIAS BIBLIOGRÁFICAS}

1. Sánchez L, Pérez J, Tamariz E, Delgado M. Seguridad del neonato hospitalizado. Aproximaciones Propuestas. Enfermería Universitaria ENEO-UNAM. 2012;9(2):27-36.

2. Hernández A, Mendoza E, Cabrera F, Ortega C. Eventos adversos en el cuidado de enfermería. Rev Mex Enf Cardiol. 2006;14 (2): 70-4.

3. Ministerio de la Protección Social. Colombia. Seguridad del Paciente. Herramientas para promover la estrategia de la Seguridad del Paciente en el Sistema Obligatorio de Garantía de Calidad de la Atención en Salud. Sistema Obligatorio de Garantia de la calidad den salud Fundación FITEC, Bogotá D.C.2007.p.14. https://www.minsalud.gov.co/sites/rid/1/ Herramientas\%20para\%20la\%20Seguridad\%20del\%20Paciente.pdf.

4. Ministerio de la Protección Social ,Colombia, Decreto número 1011de 2006, por el cual se establece el Sistema Obligatorio de Garantía de Calidad de la Atención de. de Salud. https:// www.minsalud.gov.co/Normatividad_Nuevo/DECRETO\%201011\%20DE\%202006.pdf

5. Kohn L, Corrigan J, Donaldson M, eds. To Err is Human: Building a Safer Health System. Washington, DC: Committee on Quality of Health Care in America, Institute of Medicine: National Academy Press; 2000. https://www.ncbi.nlm.nih.gov/pubmed/25077248

6. Ministerio de la Protección Social, Colombia. Resolución 1446 de 2006, por la cual se define el Sistema de Información para la Calidad y se adoptan los indicadores de monitoría del Sistema Obligatorio de Garantía de Calidad de la Atención en Salud. Disponible en:http:// www.alcaldiabogota.gov.co/sisjur/normas/Norma1.jsp?i=20267

7. Ministerio de la Protección Social, Resolución 1441 de 2013. Disponible enhttps://www. minsalud.gov.co/sites/rid/Lists/BibliotecaDigital/RIDE/DE/DIJ/resolucion-1441-de-2013.pdf

8. Raduenz A, Hoffman P, Radunz V, Marcon G, Alves I, Beryl P. Cuidado de enfermería y seguridad del paciente: visualizando la organización, acondicionamiento y distribución de 
medicamentos con método de investigación fotográfica. Rev. Latino-Am. Enfermagem. 2010;18(6):2-10.

9. Modelski A, Wegne W, Alger S, Rubim PE. Safety and protection for hospitalized children: literature review. Rev.Latino-Am. Enfermagem 2009;17(3):410-6.

10. Gómez O, Arenas W, González L, Garzón J, Mateus E, Soto A, Cultura de seguridad del paciente por personal de enfermería en Bogotá, Colombia. Cienc. Enferm, 2011;17(3):97-111.

11. Castro M, Marin H, Carvalho W. Ocorrências adversas e conseqüências imediatas para os pacientes em Unidade de Cuidados Intensivos Pediátricos. Acta Paul Enferm. 2003;16(3):62-70.

12. Fernández N. Los eventos adversos y la calidad de atención: estrategias para mejorar la seguridad de los pacientes pediátricos. Arch. argent. Pediatr 2004;102 (5):402-10.

13. Barrera-Ortiz L, Vargas-Rosero E, Cendales PA. Encuesta de caracterización para el cuidado de una persona con enfermedad crónica. Investig Enferm. Imagen Desarr. 2015;17(1):27-43.

14. Estándares de calidad de cuidados para la Seguridad del paciente en los hospitales del SNS, proyecto SENECA: informe técnico 2008. Madrid: Ministerio de Sanidad y política Social; 2009. http://www.msssi.gob.es/organizacion/sns/planCalidadSNS/docs/SENECA.pdfogle. es\&sl=pt\&u=http://www.wma. net/en/30publications/10policies/b3/index.html.

15. Declaración de Helsinki de la Asociacion Medica MundiaL Principios éticos para las investigaciones médicas en seres humanos (59a Asamblea General, Seúl, Corea, octubre 2008) Punto 32. http://www.ctomedicina.com/impugnaciones2014/bibliografiaP202MIR.pdf

16. Ministerio de Salud. Colombia. Resolución 008430 de 1993, Octubre 1993. Por el cual se establecen las normas científicas técnicas y administrativas para la investigación en salud. Bogotá D.C; Colombia. 1993. Disponible en: https://es.scribd.com/doc/49217912/ RESOLUCION-No-008430-DE-1993.

17. Moguel G, Amor-Santoyo S, Barragán-Pérez E. Seguridad de los pacientes, prioridad del Hospital Infantil de México: punto de vista del médico como paciente. Bol. Med. Hosp. Infant. Mex. 2008; 65(5): 407-11.

18. Castañeda-Hidalgo H, Garza R, González F, Pineda M, Acevedo G, Aguilera A. Percepción de la cultura de seguridad de los pacientes por el personal de enfermería. Ciencia y Enfermería. 2013;19(2):77-88.

19. Barbosa L, Cárdenas E, García L, More L, Muñoz M. Establecimiento de una línea de base de la cultura de la seguridad de los pacientes en un hospital universitario en Bogotá. Revista iberoamericana de psicología: Ciencia y tecnología. 2008;1(1):19-28.

20. Mélendez-Méndez C, Garza-Hérnandez R, González-Salinas JF, Castillo-Castillo S, GonzálezGarcía S, E Ruiz-Hernández. Percepción del personal de enfermería hacia la cultura de seguridad en un hospital pediátrico en México. Rev. Cuidarte. 2014;5(2):774-81.

21. Castillo I, Torres N, Ahumada A, Cardenas K, Licona S. Estrés laboral en enfermería y factores asociados. Cartagena (Colombia), salud uninorte. 2014;30(1):34-43.

22. Sánchez B. Cuidado seguro: la nueva tendencia en el cuidado de la salud. Actualizaciones en Enfermería, 2011;14(2):34-45.

23. Gómez O, Arenas W, González L, Garzón Jr, Mateus E, Soto A. Cultura de seguridad del paciente por personal de enfermería en Bogotá, Colombia. Cienc. enferm. 2011;17(3): 97-111.

24. Meyer G, Foster N, Christrup S, Eisenberg J. Setting a research agenda for medical errors and patient safety. Health Serv Res . 2001;36(11):5-9.

25. Organización Mundial de la Salud. Alianza Mundial para la Seguridad del Paciente [consultado 28 de febrero de 2016]. En: http://www.who.int/patientsafety/en/brochure_fin al.pdf.

26. WHO Patient Safety Curriculum Guide for Medical Schools, Evaluation Study. World Health Organization, Geneva. 2011. Available: http://www.who.int/patientsafety/education/ curriculum/PSP_Eval_Study_Report-2011_March-2012.pdf.

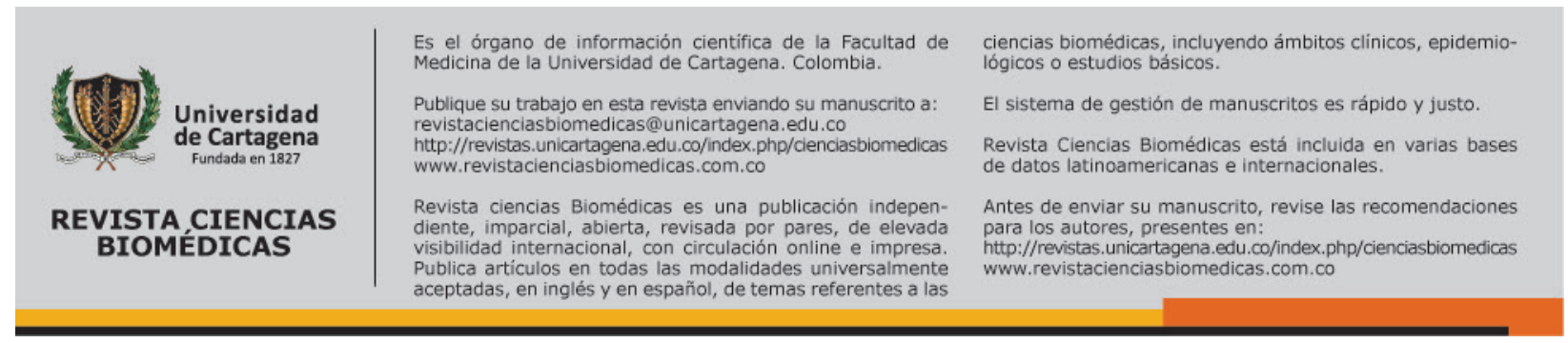

